(Aus dem physiologischen Institut der Universität Wien.)

\title{
Zur Kenntnis der Geschlechtsbestimmung beim Menschen.
}

Vou

\section{Felieie Ewart.}

Wie mir Prof. Sigm. Ex ner mitteilte, erweckten Erfahrungen in verwandten und befreundeten Familien in ihm die Vermutung, dass bei der Geburt eines zweiten, resp. dritten oder vierten Kindes die Wahrscheinlichkeit grösser sei, dass dessen Geschlecht mit dem des vorhergehend geborenen übereinstimme, als dass es wechsle. Doch war ihm das nur eine Vermutung, an die sich der allgemeinere Gedanke knüpfen liess, ob wohl die Faktoren, welche das Geschlecht eines Kindes bestimmen, ganz unabhängig sind von jenen, die für das Geschlecht eines vorhergehenden oder nachfolgenden Kindes derselben Eltern maassgebend waren oder sein werden. Er dachte daran, aus den Taufbüchern eines orler mehrerer ländlicher Pfarrämter grössere Zahlen zu gewinnen, da hier die Bewegung der Bevölkerung eine geringere ist als in den Städten. Dieser Plan stiess auf Schwierigkeiten, bildete aber die Anregung zu der nachfolgenden Untersuchung.

Nachdem ich mich entschlossen hatte, sie durchzuführen, schien mir nach einer genauen Überlegung, dass für den vorliegenden Zweck die Verwendung des Gothaschen Kalenders zulässig wäre, und bei der Reichhaltigkeit seines Materials, der Bequemlichkeit der Handhabung, der allgemein zugänglichen Kontrollierbarkeit und wohl auch in mancher anderen Beziehung grosse Vorteile gegenüber einem lokal gesammelten Material bieten würde.

Ich bin mir dessen wohl bewusst, dass auch im Gothaschen Kalender manche Lücke enthalten und manche falsche Angabe verzeichnet sein wird. Bei den uns beschäftigenden Fragen, deren Beantwortung sich sämtlich auf grosse Zahlen stützt, handelt es sich aber nicht um die absolute Fehlerfreiheit des zugrunde gelegten 
Materials, sondern ausschliesslich darum, ob die vorhandenen Fehler irgendeinen maassgebenden Einfluss auf die Zahlenresultate ausüben können, die als Basis für die Schlussfolgerungen zu dienen haben. Mit anderen Worten, man wird nur jene gewonnenen Zahlenverhältnisse als richtig annehmen dürfen, auf welche die voraussichtlich vorkommenden Fehler des statistischen Materials (nicht notierte Tod- oder Fehlgeburten, falsche Paternitätsangaben usw.) prinzipiell keinen oder nur einen verschwindendien Einfluss haben. So wie z. B. die Verhältniszahl zwischen männlichen und weiblichen Geburten durch die Ausschaltung der Fehlgeburten nicht nennenswert beeinflusst wird, so gibt es noch manche andere Grössen, die mit jenen nicht in funktionellem Verhältnis stehen.

Es schien mir überflüssig, alle drei Jahresbände des Gothaschen Kalenders für meine Studien zu verwenden, und so griff ich denn auf das Geratewohl nach dem Jahrgang 1900 des Gothaschen genealogischen Hofkalenders ${ }^{1}$ ) und habe daraus von Anfang beginnend 553 Familien mit 2500 Geburten und 1010 gleichen Geschlechtsfolgen als Basis meiner Untersuchung verwertet. Von gleicher Geschlechtsfolge spreche ich, wenn zwei aufeinanderfolgende Geburten Kinder desselben Geschlechtes ergeben.

Ich babe natürlich nur Familien, die mehr als ein Kind aufweisen, berücksichtigt, und die wenigen übersprungen, bei denen aus mir unbekannten Gründen die Angaben, die ich für meine alsbald zu beschreibenden Tabellen brauchte, nicht vollständig waren (Angabe des Geburtstages usw.). Da ich nämlich die Hoffnung hegte, auch noch andere Beziehungen statistisch festlegen zu können, so habe ich mir das Material in Tabellen zusammengestellt, aus welchen nicht nur die Geschlechtsfolgen, sondern auch noch andere Daten zu entnehmen sind, so dass diese Tabellen eine Form erhielten, wie sie das vorliegende Beispiel für eine Familie zeigt.

1) 137. Jahrgang, Justus Perthes. Gotha.

2) Nach Abschluss meiner Arbeit bemerke ich, dass schon M. W e inberg in einer Abhandlung (Methode und Ergebnisse der Erforschungen der Ursachen der Mehrlingsgeburten. Virchow's Arch. Bd. 171 zitiert nach Rosenfeld, Zeitschr. f. Geburtshilfe u. Gynäkologie Bd. 50 S. 30) einen ähnlichen Weg eingeschlagen hat, um statistisches Material für Geburtsfälle zu gewinnen. Er benutzte dazu nicht den Gothaschen Kalender, sondern das drei Bände umfassende Jahrbuch des deutschen Adels, welches 216 Adelsgeschlechter mit 5432 verheirateten Männern behandelt. 
Tabelle I.

\begin{tabular}{|c|c|c|c|c|c|c|c|c|c|c|c|c|c|c|c|c|}
\hline \multirow{2}{*}{$\begin{array}{l}\text { Name } \\
\text { der } \\
\text { Familie }\end{array}$} & \multicolumn{3}{|c|}{$\begin{array}{c}\text { Zahl } \\
\text { der } \\
\text { Kinder }\end{array}$} & \multicolumn{2}{|c|}{$\begin{array}{c}\text { Erst- } \\
\text { ge- } \\
\text { burt }\end{array}$} & \multicolumn{3}{|c|}{$\begin{array}{c}\text { Gleiche } \\
\text { Ge- } \\
\text { schlechts- } \\
\text { folgen }\end{array}$} & \multirow{2}{*}{ 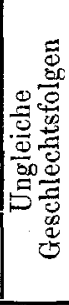 } & \multicolumn{2}{|c|}{$\begin{array}{c}\text { Monate } \\
\text { zwischen } \\
\text { gleichen Ge- } \\
\text { schlechts- } \\
\text { folgen }\end{array}$} & \multirow{2}{*}{ 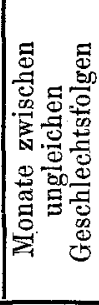 } & \multirow{2}{*}{ 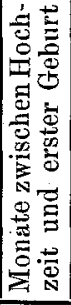 } & \multirow{2}{*}{ 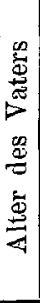 } & \multirow{2}{*}{ 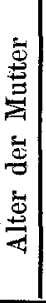 } & \multirow{2}{*}{ 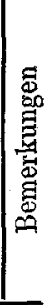 } \\
\hline & : & 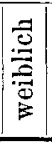 & $\underset{\Xi}{\stackrel{\Xi}{\Xi}}$ & 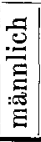 & : & : & \begin{tabular}{l}
$\frac{5}{0}$ \\
\hdashline \\
0 \\
0 \\
0
\end{tabular} & 沾 & & 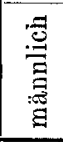 & 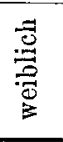 & & & & & \\
\hline & 5 & 1 & 6 & 1 & 0 & 3 & 0 & 3 & 2 & 88 & 0 & 40 & 26 & 25 & 21 & \\
\hline
\end{tabular}

$\mathrm{Zu}$ derselben wäre nur zu bemerken, dass ich die Zeit zwischen den Geburten zu ganzen Monaten abgerundet habe, so dass die einzelnen Angaben um böchstens 15 Tage von der Wahrheit abweichen. Ähnlich verfuhr ich in bezug auf die Zahlen, welche die Lebensjahre der Eltern angeben, so dass hier die Abweichungen von der Wahrheit im maximum 6 Monate betragen. Findet sich im Gothaschen Kalender die Angabe, dass das Familienoberhaupt eine zweite Ehe eingegangen ist, so wurde diese in einer selbständigen Tabelle notiert.

Bei Zwillingsgeburten, von denen in meinen Tabellen 21 verzeichnet sind, habe ich gleichgeschlechtliche als gleiche, ungleichgeschlechtliche als ungleiche Geschlechtsfolgen aufgefasst.

\section{Wahrscheinlichkeit gleicher Geschlechtsfolgen.}

Bei den 2500 geprüften Geburten ergaben sich 1010 gleiche Geschlechtsfolgen, und zwar 506 männliche und 504 weibliche, während der Wechsel im Geschlecht 897 mal vorkam.

Es fragt sich nun zunächst, ob die Differenz von 1010 gleichen und 897 ungleichen Geschlechtsfolgen der Ausdruck der unvermeidlichen Zufälligkeiten einer derartigen Statistik ist und verschwinden würde, wenn die Zahl der Fälle grösser und grösser gewählt worden wäre, oder ob sich in dieser Differenz, welche recht bedeutend ist und über $10 \%$ der ungleichen Geburtsfolgen beträgt, eine Gesetzmässigkeit ausspricht. Ausser dieser ersten Möglichkeit, dass man es wegen einer zu geringen Zahl von Fällen mit dem Ausdruck der Zufälligkeit zu tun hat, besteht noch eine zweite, gegeben durch die bekannte Tatsache der ungleichen Zahlen männlicher und weiblicher Geburten. Diese Tatsache allein lässt schon von vorne herein eine 
Differenz zwischen den gleichen und ungleichen Geschlechtsfolgen erwarten.

Um die erste Möglichkeit auf ihr Zutreffen zu prüfen, habe jch der Reihe nach das ganze Material der 1010 gleichen Geschlechtsfolgen geteilt in je zehn Abteilungen à 100 und einer à 10 Folgen, und für jede dieser elf Reihen die Anzahl der ungleichen Geschlechtsfolgen bestimmt (vergl. Tab. II).

Tabelle II.

\begin{tabular}{c|c|c}
\hline & \multicolumn{2}{|c}{ Es kommen: } \\
\hline in Gruppe & $\begin{array}{c}\text { auf gleiche } \\
\text { Geschlechtsfolgen }\end{array}$ & $\begin{array}{c}\text { ungleiche } \\
\text { Geschlechtsfolgen }\end{array}$ \\
\hline & 100 & 82 \\
I & 100 & 68 \\
III & 100 & 77 \\
IV & 100 & 74 \\
V & 100 & 98 \\
VI & 100 & 101 \\
VII & 100 & 97 \\
VIII & 100 & 99 \\
IX & 100 & 106 \\
X & 100 & 80 \\
XI & 10 & 15 \\
\hline- & 1010 & 897
\end{tabular}

Man ersieht hieraus, dass in den elf Reihen nur dreimal die Zahl der ungleichen Geschlechtsfolgen grösser ist als die der gleichen, wobei die letzte Reihe von uur zehn Posten kaum in Betracht gezogen werden kann, bei den beiden anderen Fällen aber ein Plus von nur 1 und von 6 vorhanden ist, gegenüber dem Minus von 18, $32,23,26,2,3,1,20$ in den anderen Reihen. Unter diesen Umständen wird man die auffallende Tatsache, dass von sämtlichen Geburtsfolgen 5,9\% mehr gleichgeschlechtliche als ungleichgeschlechtliche vorhanden sind, nicht für den Ausdruck von Zufälligkeiten halten dürfen. Um mich von solchen noch unabhängiger zu machen, habe ich die Rechnung weiterhin in der Form ausgeführt, dass ich die Serie von 2500 Geburten in 25 gleiche Teile ${ }^{1}$ ) geteilt habe, und für

1) Da die Anzahl von 100 Kindern nicht immer mit den Familien, wie sie in meinen Tabellen der Reihe nach standen, zusammenfielen, so habe ich mir auf folgende Weise dabei geholfen. Ich zählte der Reihenfolge nach und suchte, um das Hundert schliesslich voll zu machen, eine Familie mit der notwendigen Kinderanzahl heraus. Beim nächsten Hundert wurden die übersprungenen Familien zuerst gerechnet und dann in der beschriebenen Weise fortgefahren. 
jede Abteilung, bestehend aus je 100 Geburten, die Anzahl der gleichen und die der ungleichen Geschlechtsfolgen notiert habe. Dies ergab folgende Tabelle:

Tabelle III.

\begin{tabular}{|c|c|c|}
\hline \multicolumn{3}{|c|}{ Es kommen anf je 100 Geburten: } \\
\hline in Gruppe & $\begin{array}{c}\text { gleiche } \\
\text { Geschlechtsfolgen }\end{array}$ & $\begin{array}{c}\text { ungleiche } \\
\text { Geschlechtsfolgen }\end{array}$ \\
\hline $\begin{array}{r}I \\
I I \\
\text { III } \\
\text { IV } \\
\text { V } \\
\text { VI } \\
\text { VII } \\
\text { VIII } \\
\text { IX } \\
\text { X } \\
\text { XI } \\
\text { XII } \\
\text { XIII } \\
\text { XIV } \\
\text { XV } \\
\text { XVI } \\
\text { XVII } \\
\text { XVIII } \\
\text { XIX } \\
\text { XX } \\
\text { XXI } \\
\text { XXII } \\
\text { XXIII } \\
\text { XXIV } \\
\text { XXV }\end{array}$ & $\begin{array}{l}48 \\
42 \\
44 \\
44 \\
47 \\
46 \\
38 \\
43 \\
46 \\
44 \\
39 \\
41 \\
38 \\
33 \\
33 \\
38 \\
41 \\
42 \\
32 \\
44 \\
28 \\
42 \\
35 \\
38 \\
44\end{array}$ & $\begin{array}{l}31 \\
38 \\
33 \\
33 \\
25 \\
31 \\
45 \\
37 \\
30 \\
40 \\
38 \\
38 \\
36 \\
32 \\
38 \\
39 \\
38 \\
39 \\
42 \\
29 \\
47 \\
33 \\
37 \\
37 \\
31\end{array}$ \\
\hline- & 1010 & 897 \\
\hline
\end{tabular}

Auch bei dieser Zusammenstellung sieht man, dass in den 25 Serien à 100 Geburten nur sechsmal die gleichen Geschlechtsfolgen kleiner als die ungleichen sind, gegenüber dem umgekehrten Fall in 19 Serien.

Also auch wenn man die Fälle zu kleineren Gruppen zusammenstellt, sprechen die Resultate für die Gesetzmässigkeit des Überwiegens gleicher Geburtsfolgen.

Die zweite Möglichkeit einer Erklärung für die geschilderte Tatsache liegt wie gesagt in dem Umstande, dass, wie allgemeine Statistiken ergaben, mehr Knaben als Mädchen geboren werden, wodurch allein schon eine Differenz in den gleichen und ungleichen Geschlechtsfolgen bedingt sein muss.

Sehen wir nach, wie gross nach der Wahrscheinlichkeitsrechnung: diese Differenz sein muss. 
Es werden wie angegeben wird auf 100 Mädchen 106,3 Knaben geboren 1).

Dementsprechend können wir uns vorstellen, es befänden sich in einer Urne 100 weisse und 106,3 schwarze Kugeln; es würde blind je eine Kugel aus der Urne genommen, nachher wieder hinein geworfen, neuerdings eine genommen usw., und es fragt sich nun, wie oft werden nacheinander zwei gleiche, wie oft werden zwei ungleiche Kugeln gezogen. Es sind folgende vier Fälle möglich:

$\alpha$ ) Dass die I. Kugel weiss ist, hat die Wahrscheinlichkeit $\frac{100}{206,3}$, dass die II. Kugel weiss ist, hat abermals die Wahrscheinlichkeit $\frac{100}{206,3}$, somit dass beide Kugeln weiss, sind die Wahrscheinlichkeit $\frac{100^{2}}{206,3^{2}}$.

$\beta$ ) Dass die I. Kugel schwarz ist, hat die Wahrscheinlichkeit $\frac{106,3}{206,3}$, dass die II. Kugel schwarz ist, hat dieselbe Wahrscheinlichkeit $\frac{106,3}{206,3}$, somit dass I. und II. Kugel schwarz sind, die Wahrscheinlichkeit $\frac{106,3^{2}}{206,3^{2}}$.

In diesen beiden Fällen herrscht also gleiche Folge.

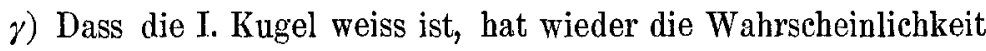
$\frac{100}{206,3}$, dass die II. Kugel schwarz ist, die Wahrscheinlichkeit $\frac{106,3}{206,3}$, somit die Wahrscheinlichkeit für diese Folge gleich $\frac{100 \times 106,3}{206,3^{2}}$.

d) Dass die I. Kugel schwarz ist, hat die Wahrscheinlichkeit $\frac{106,3}{206,3}$, dass die II. Kugel weiss ist, hat die Wahrscheinlichkeit $\frac{100}{206,3}$, somit die Wahrscheinlichkeit, dass die I. Kugel schwarz und die II. Kugel weiss ist, $\frac{100 \times 106,3}{206,3^{2}}$.

Die beiden Fälle $\gamma$ und $\delta$ entsprechen den ungleichen Gesehlechtsfolgen.

1) Zitiert nach Hensen, L. Hermann's Handb. d, Physiol. Bd. 6 S. 205. 
Somit ist die Wahrscheinlichkeit der gleichen Folgen

$$
\frac{100^{2}+106,3^{2}}{206,3^{2}}=\frac{21299,69}{206,3^{2}}
$$

und die Wahrscheinlichkeit der ungleichen Folgen

$$
\frac{10630+10630}{206,3^{2}}=\frac{21260}{206,3^{2}} \text {. }
$$

Also das Verhältnis zwischen gleichen und ungleichen Folgen $\frac{21299,69}{21260}=1,00185$.

Da in meiner Statistik das Verhältnis der gleichen und ungleichen Geschlechtsfolgen $\frac{1010}{897}=1,137$ ist, so hätten von den 1907 Geschlechtsfolgen nur 954 gleiche, d. h. um 56 weniger, hingegen ebensoviel mehr ungleiche Geschlechtsfolgen gefunden werden müssen, wenn jene Ungleichheit der Geburtsfolgen nur auf der Zahlendifferenz männlicher und weiblicher Geburten beruhen würde.

Nun könnte man einwenden, dass ich nicht das aus Tausenden anderer Geburtsfälle entnommene Verhältnis von 100 weiblichen zu 106,3 männlichen Kindern zur vorstehenden Berechnung der Wahrscheinlichkeit gleicher und ungleicher Geburtsfolgen hätte verwenden sollen, sondern die Geburtsfälle meines eigenen Materials. Der Zufall wollte es, dass in diesem 1273 männliche auf 1227 weibliche Kinder entfallen, hier also das Verhältnis

$$
w: m=100: 103,7
$$

besteht, so dass die Wahrscheinlichkeit gleicher Geburtsfolgen noch bedeutend kleiner ist als bei dem meiner Rechnung zugrunde gelegten Verhältnisse. Somit zeigt meine Rechnung a fortiori, dass die Zahlendifferenz zwischen männlichen und weiblichen Geburten keine ausreichende Erklärung des Überwiegens gleicher Geschlechtsfolgen abgibt. Auch die Art, wie ich die Zwillingsgeburten in meine Statistik einführte, kann das erhaltene Resultat nicht erklären, denn es waren nur 21, kämen also kaum in Betracht, selbst wenn sie alle gleichgeschlechtliche gewesen wären.

Die vorgeführten Tatsachen und die daran geknüpften Betrachtungen scheinen mir vielmehr zu der Behauptung zu drängen: es bestehe irgendein Faktor, der für die Bestimmung des Geschlechtes eines Kindes yon Bedeutung ist, und der in gleicher oder einer geringeren Grösse noch fort- 
wirkt, weinn das Geschlecht des nächstfolgenden Kindes bestimmt wird.

Diesen Faktor wird man wohl in den Eltern zu suchen haben; vielleicht in der Mutter, vielleicht im Vater, vielleicht im Verhältnis beider zueinander.

Ist derselbe in seiner Grösse abhängig von der Zeit, so muss sich auch das aus meiner Statistik ergeben. Ich habe deshalb die durchschnittliche Anzahl der Monate berechnet, welche verfliessen zwischen zwei gleichgeschlechtlichen Geburten und zwei ungleich geschlechtlichen Geburten.

Meine Tabellen enthalten in dieser Beziehung Angaben, die in den Zahlen der folgenden Tabelle IV zusammengestellt sind.

Tabelle IV.

\begin{tabular}{l|c|c|c}
\hline Geburten & $\begin{array}{c}\text { Zahl der } \\
\text { Geburtsfolgen }\end{array}$ & $\begin{array}{c}\text { Gesamtdifferenz } \\
\text { in Monaten }\end{array}$ & $\begin{array}{c}\text { Durchschnittliche } \\
\text { Zeitdifferenz } \\
\text { in Monaten }\end{array}$ \\
\hline Gleichgeschlechtliche . . & 1010 & $\begin{array}{l}30266 \\
29473\end{array}$ & $\begin{array}{c}29,97 \\
32,86\end{array}$
\end{tabular}

Man sieht somit eine durchschnittliche Zeitdifferenz von fast 3 Monaten zwischen gleich- und ungleichgeschlechtlichen Geburtsfolgen, und es drängt sich wieder die Frage auf, ob dies zufällig sei oder nicht. Auch hier können wir versuchen, diese Frage nach der oben angewendeten Methode zu beantworten; rechnen wir dieselben Daten für die elf Serien, von denen die ersten zehn 100 gleiche, die elfte zehn gleiche Geburtsfolgen enthält, so gewinnen wir die folgende Tabelle V.

$$
\text { Tabelle V. }
$$

\begin{tabular}{c|c|c|c|c}
\hline \multirow{2}{*}{$\begin{array}{c}\text { Nummer } \\
\text { der Serien }\end{array}$} & \multicolumn{2}{|c|}{$\begin{array}{c}\text { Summe der Zeitdifferenz } \\
\text { im Monaten }\end{array}$} & \multicolumn{2}{|c}{ Durchschnitt in Monaten } \\
\cline { 2 - 5 } & $\begin{array}{c}\text { gleiche } \\
\text { Geburtsfolgen }\end{array}$ & $\begin{array}{c}\text { ungleiche } \\
\text { Geburtsfolgen }\end{array}$ & $\begin{array}{c}\text { gleiche } \\
\text { Geburtsfolgen }\end{array}$ & $\begin{array}{c}\text { ungleiche } \\
\text { Geburtsfolgen }\end{array}$ \\
\hline & 3137 & 2303 & 31,37 & 28,09 \\
II & 2885 & 2545 & 28,85 & 37,43 \\
III & 3026 & 2066 & 30,26 & 27,55 \\
IV & 2925 & 2298 & 29,25 & 30,24 \\
VI & 2852 & 3248 & 28,52 & 33,48 \\
VI & 2863 & 3845 & 28,63 & 37,70 \\
VII & 2942 & 3191 & 29,42 & 32,90 \\
VIII & 3023 & 2969 & 30,23 & 29,99 \\
IX & 3007 & 3667 & 30,07 & 36,31 \\
X & 3284 & 2710 & 32,84 & 31,88 \\
XI & 138 & 631 & 13,8 & 42,07
\end{tabular}


Man erkennt aus derselben, dass nur in vier von den elf Serien die durchschnittliche Pause zwischen zwei ungleichen Geburtsfolgen kleiner ist als bei zwei gleichen Geburtsfolgen, so dass auch diese elf Serien durch ihr Verhältnis $7: 4$ auf eine Abhängigkeit der Wahrscheinlichkeit gleicher Geburtsfolgen von der Grösse der Pause zwischen den Geburten hindeuten.

Es wird also wohl so sein, dass die Faktoren, welche das Geschlecht eines Kindes bestimmen, durch Monate und Jahre weiter wirken, wenn auch nur in so geringem Grade, dass sie neben anderen Faktoren unscheinbar werden und nur durch derartige statistische Feststellungen nachgewiesen werden können.

Ich habe das Problem auch noch in einer dritten Form zu beantworten gesucht. Teilt man nämlich die sämtlichen Geburtsfolgen meines Materiales nach solchen, in welchen zwei Kinder derselben Ehe innerhalb 20 Monaten geboren wurden, und solchen, in denen die Geburten weiter als 20 Monate anseinander lagen, so findet man das folgende Resultat.

In der ersten Gruppe sind:

397 gleiche Geburtsfolgen,

343 ungleiche Geburtsfolgen.

In der zweiten Gruppe sind:

613 gleiche Geburtsfolgen,

554 ungleiche Geburtsfolgen.

Somit ergeben die zeitlich nahe aneinander liegenden Geburten: $53,65 \%$,

die zeitlich ferner liegenden Geburten

gleicher Geburtsfolgen.

$$
52,53 \%
$$

Wie man sieht, kein bedeutender, aber doch merklicher Unterschied zugunsten der eben aufgestellten These.

A. Geissler ${ }^{1}$ ) hat an einem ungemein reichen Material, den amtlichen Geburtszählkarten des Königreichs Sachsen, die einen Zeitraum von 10 Jahren umfassen, nebst mehreren anderen sehr interessanten Fragen auch die der Geschlechtsfolgen teilweise beantwortet.

1) A. Geissler, Beiträge zur Frage des Geschlechtsverhältnisses der Geborenen. Zeitschr. d. k, sächsischen statistischen Bureaus Jahrg. 1889. 
Er kommt nämlich unter anderem zu dem Resultat, dass im Fall einer männlichen Erstgeburt die Aussicht auf eine Geschlechtswiederholung nicht unerheblich grösser ist, als wenn die Erstgeburt ein Mädchen war. Doch lässt sich kein Einfluss der Erstgeburt auf das Geschlecht aller nachfolgenden Geburten nachweisen.

Eine weitere Frage, welche nahe lag, und die ich an meinem Material leicht beantworten konnte, lautet: Hat das Altersverhältnis zwischen Vater und Mutter einen merklichen Einfluss auf das zu erwartende Kind? Ich habe deshalb in meinen Tabellen jene Familien herausgesucht, bei denen der Vater um 10 Jahre älter ist als die Mutter, und deren Kinder nach dem Geschlechte gruppiert. Dasselbe tat ich bei allen Familien, deren Eltern keinen grösseren Altersunterschied als 10 Jabre aufwiesen. Es hat sich dabei ergeben, dass die Familien der ersten Gruppe 594 Kinder, darunter 287 Knaben aufwiesen; die Familien der zweiten Gruppe unter 1906 Kindern 990 Knaben besassen; das beisst die ersteren haben nur 48,32\% Knaben, die letzteren $51,94 \%$ männliche Nachkommen. Ich kann dieses Resultat um so weniger für Zufall halten, als in meiner Statistik das Verhältnis zwischen Knaben und Mädchen 103,7 : 100 ist. Hiernach hätte man erwarten sollen, dass auf die 307 Töchter der älteren Väter 318,36 Söhne entfallen, während tatsächlich um 31,36 weniger vorhanden sind. Oder anders ausgedrückt, während in dem genannten Zahlenmaterial nur 3,7\% mehr männlicbe Geburten enthalten sind, weist die Nachkommenschaft der alten Väter um 3,62\% weniger Knaben auf, was zusammen eine Differenz von $7,32 \%$ zu ungunsten der männlichen Geburten bedeutet.

In dem von $\mathrm{Hensen}$ bearbeiteten Abschnitt über Zeugung in Hermann's Handbuch der Physiologie findet sich Bd. 6 S. 206 eine Tabelle, in welcher der Altersunterschied zwischen den Eltern und die Zahl der aus diesen Ehen hervorgegangenen männlichen Geburten in Beziehung zueinander gebracht sind. Lin Blick auf diese Tabelle zeigt, dass ein nennenswertes Überwiegen der Knabengeburten dann stattfindet, wenn der Vater älter ist als die Mutter, im Gegensatz zu dem Falle, dass die Eltern im gleichen Alter stehen. Mein Resultat weicht also offenbar von diesem deshalb ab, weil die Väter meiner Statistik nicht nur, wie es der Norm entspricht, älter als die Mütter, sondern um mehr als 10 Jahre älter waren. 
$\mathrm{Kisch}{ }^{1}$ ) hat eine ähnliche Untersuchung, auch mit Hilfe der Angaben des Gothaer Kalenders, aber wie aus seinen Worten (Wiener Klinik S. 222) hervorgeht, auf Grundlage anderer Familien angestellt, und stützt seine Schlüsse auf 556 Ehen mit 1972 Geburten. Er hat auch das Alter der Mutter genauer berücksichtigt und kommt zu folgender These: "Wenn der Mann mindestens um 10 Jahre älter ist als die Frau, und diese sich in den Jahren der höchsten Reproduktionsfähigkeit befindet (d. i. 20-25 Jahre alt ist), so entstehen ganz bedeutend mehr Knaben als Mädchen."

Auch Väter, die um mehr als 10 Jahre älter sind als ihre Frauen, erzeugen mehr Knaben als Mädchen, während, wenn Mann und Frau g]eich alt sind, sich ein Mädchenüberschuss ergibt. Sind die Frauen älter als die Männer, so findet sich ein mässiger Knabenüberschuss. K i s ch meint, dass die Zahlen, über die er verfügt, nicht genügend gross sind, um endgültige Schlüsse daraus zu ziehen. Diese Überlegung trifft wohl auch mein Material.

Endlich interessierte es mich noch nachzusehen, wie sich die Erstgeburten in bezug auf ihr Geschlecht zu der Gesamtzahl der Kinder verhalten. In den 571 Ehen meiner Tabellen hatten die Erstgeborenen 320 mal männliches, 251 mal weibliches Geschlecht; somit kamen auf 100 Mädchen 127,49 Knaben. Da auf 100 Mädchen der gesamten Familien 103,7 Knaben entfallen, so ergibt das für die Erstgeborenen einen Überschuss von 23,79, was man auch kaum für einen Zufall halten wird. Es scheint deshalb die Wahrscheinlichkeit, dass die Erstgeburt ein Knabe ist, recht bedeutend zu überwiegen.

Herr Hofrat Sigm. Exner möge an dieser Stelle meinen ergebensten Dank für die mir gewährte Anregung und Unterstützung gütigst entgegennehmen.

1) E. H. Kisch, Über den gegenwärtigen Standpunkt der Lehre von der Entstehung des Geschlechtes beim Menschen. Wiener Klinik 1887. - E. H. Kisch, Zur Geschlechtsentstehung beim Menschen. Autoreferat $\mathrm{Humboldt} 1888$ S. 297. 